\title{
Myelofibrosis involving lymph node: a novel cytogenetic abnormality in a mimicker of mesenchymal neoplasm
}

\author{
Youjun Hu • Ann-Leslie Zaslav • \\ Neetu Radhakrishnan • Marc Golightly • \\ Colette Pameijer
}

Received: 13 May 2009 / Accepted: 10 June 2009/Published online: 30 June 2009

(C) Springer-Verlag 2009

\begin{abstract}
A case of primary myelofibrosis involving lymph node and with a novel cytogenetic abnormality [del (18) (p11.2-3)] is reported. The abnormalities are identical among specimens from the lymph node, peripheral blood, and bone marrow that were analyzed years apart. Additionally, we show that the infiltrate by dysplastic megakaryocytes in the lymph node morphologically mimics a metastatic mesenchymal neoplasm, even when the clinical history myelofibrosis was known.
\end{abstract}

Keywords Megakaryocyte $\cdot$ Myelofibrosis ·

Immunohistochemical stain $\cdot$ Cytogenetics

\section{Introduction}

Primary myelofibrosis (PMF) is a rare chronic myeloproliferative disorder that usually involves the bone marrow with proliferation of megakaryocytes and granulocytic elements and has a variable degree of marrow fibrosis (1). When the marrow is fibrotic, there is usually myeloid metaplasia or extramedullary hematopoiesis that involves most commonly

Y. Hu $(\bowtie) \cdot$ A.-L. Zaslav $\cdot$ M. Golightly

Department of Pathology, Stony Brook University Hospital,

Stony Brook, NY 11794, USA

e-mail: yohu@notes.cc.sunysb.edu

N. Radhakrishnan

Hematology \& Oncology, Department of Internal Medicine,

Stony Brook University Hospital,

Stony Brook, NY 11794, USA

C. Pameijer

Department of Surgery, Stony Brook University Hospital,

Stony Brook, NY 11794, USA the spleen and liver. Other organs occasionally may be involved as well, such as lymph node, breast, skin, urinary bladder, etc.(2). The exact molecular mechanism of myelofibrosis is not clear. No specific recurrent cytogenetic abnormalities have been described. Diagnosis of extramedullary hematopoiesis involving lymph nodes or other organs is usually straightforward, given the appropriate clinical setting. Here, we report a case of myelofibrosis in which the lymph node is infiltrated with dysplastic megakaryocytes along with fibrosis. The cytogenetic abnormality in the lymph node was the same as that identified in the marrow 4 years prior. The morphological changes raised the possible differential diagnosis of metastatic mesenchymal neoplasm, even though the clinical history of myelofibrosis was known.

\section{Clinical course}

The patient is a 71-year-old female who was diagnosed with primary myelofibrosis 16 years ago when she presented with anemia and splenomegaly. Four years ago, the patient became transfusion-dependent. The marrow was fibrotic with clusters of dysplastic megakaryocytes and largely diminished normal hematopoietic elements. Her transfusion requirements decreased once she was started on thalidomide and prednisone with anagrelide and hydroxyurea for thrombocytosis. After approximately 1 year, she again became transfusiondependent and continued on only hydroxyurea for thrombocytosis. Last year, the hydroxyurea was discontinued after she received splenic radiation for symptomatic splenomegaly. The patient developed secondary hemochromatosis and was placed on iron chelation therapy. Ten months ago, her spleen was again irradiated for symptomatic splenomegaly. Subsequently, the patient presented with congestive heart failure due to severe pulmonary hypertension of unclear etiology. 
Workup for a pulmonary embolism, extramedullary pulmonary infiltrates, or valvular or ischemic heart diseases was negative. She was started on sildenafil for pulmonary hypertension and hypoxia. The patient has been on erythropoietin supplementation for 2 years. Her physical exam was remarkable for a hard, mobile right axillary node $2 \times 1 \mathrm{~cm}$ in size. There was moderate to massive splenomegaly and hepatomegaly. No focal lesions were found on an ultrasound of the liver. The complete blood cell count showed mild leukopenia, anemia, and a platelet count of $360 \times 10^{3} / \mu 1$. Circulating blasts were $2 \%$ (stable for the last 6 months). Test for JAK2 mutation was negative. Biopsy of the enlarged axillary lymph node was performed.

\section{Materials and methods}

Histologic and immunohistochemical studies

The specimen was processed following routine histologic procedures including routine H\&E stain and immunohistochemical stains. The antibodies were from Dako (antibodies Vimentin, CD3, CD20 and CD45, Carpinteria, CA, USA) and Invitrogen (antibodies CD61 and Cytokeratin AE1/ AE3, Carlsbad, CA, USA). The automatic immunostainer was Bond-maX (Leica Microsystems, Australia).
Flow cytometry study

A portion of fresh tissue was sent for flow cytometry analysis. Analysis was performed on FACScalibur 2000 from Becton Dickinson (San Jose, CA, USA). The antibodies (BCL2, CD3, CD4, CD5, CD7, CD8, CD10, CD14, CD19, CD20, CD21, CD22, CD23, CD30, CD33, CD34, CD38, CD45, CD56, Cytokeratin, EpCAM, FMC7, Glycophorin, HLA-DR, Kappa light chain, Lambda light chain) were purchased from the same company. CD43 was from Caltag, Carlsbad, CA, USA.

\section{Cytogenetics study}

Cytogenetic G-banded metaphase analysis and fluorescence in situ hybridization (FISH) were performed using standard cytogenetic techniques on bone marrow (BM), the lymph node biopsy, and unstimulated blood (UB). Analyses were performed four different times during the course of treatment.

For FISH, 200 nuclei were scored per probe. The following probes were used: 6/25/07 (UB specimen): LSI 5q EGR1/ D5S23 (5q31,5p15.2), the LSI D7S486/CEP 7 (7q31/7p11.1q11.1(D7Z1)), the LSI CEP 8 (8p11.1-q11.1(D8Z2)) and the LSI 21 (21q22.13-q22.2) probes; 1/4/08 (UB specimen): all of the previous probes and the LSI MLL DC BAR (11q23) probe; 8/20/08 (lymph node specimen): 18p11.3 (D18S552)
Fig. 1 a Interphase nuclei probed with the TelVysion CEP 18 (Aqua) centromere probe and the $18 \mathrm{p}$ (yellow) probes (Abbott Mol, Inc). Presence of only one yellow signal indicates loss of one $18 \mathrm{p}(11.3)$. The right nucleus indicates the presence of both $18 \mathrm{p}$ signals (i.e., normal). b G-banded karyotype demonstrating the deleted chromosome $18 \mathrm{p}$ (11.2). The arrow indicated the deleted $18 \mathrm{p}$ homolog
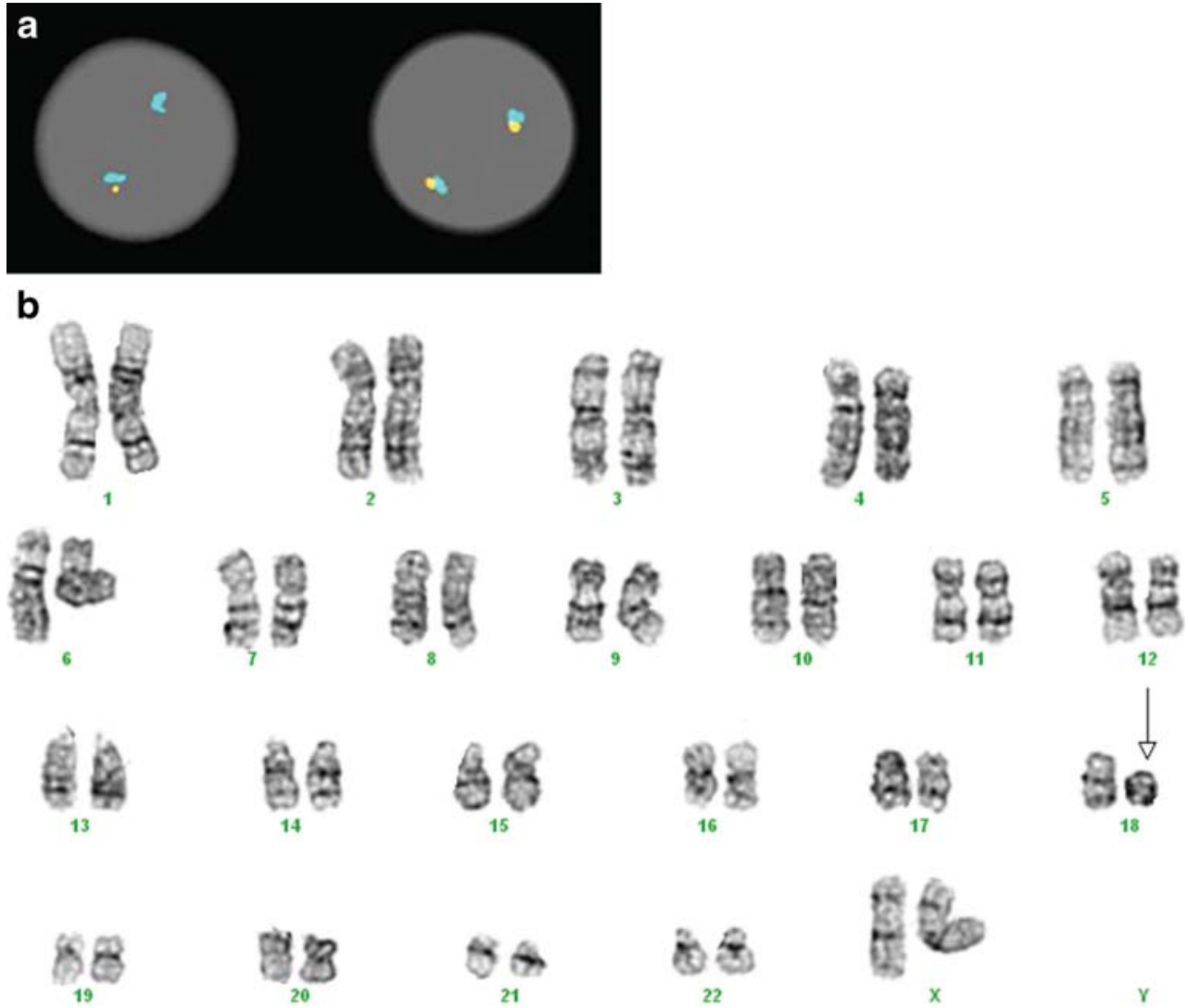


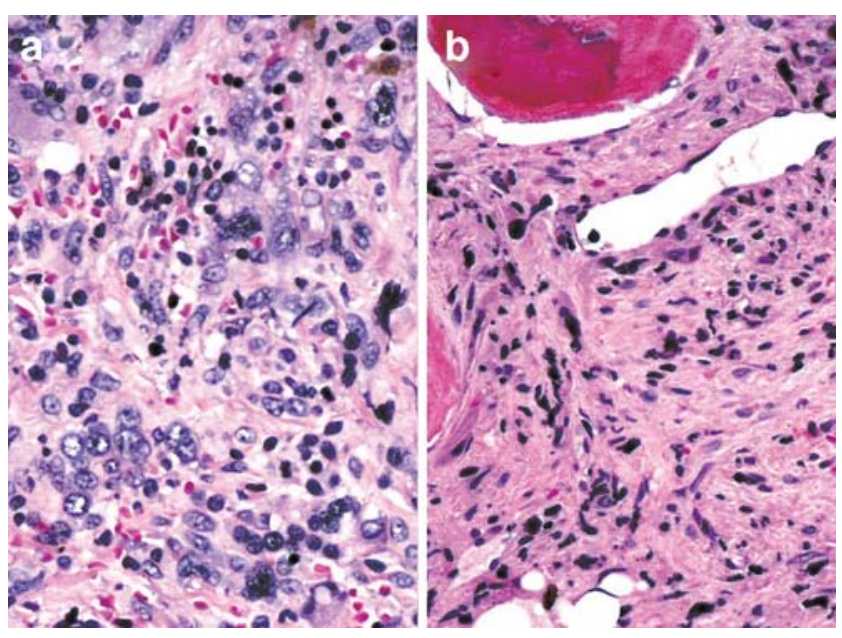

Fig. 2 a Lymph node biopsy, H\&E stain, $\times 200$ magnification. b Bone marrow core biopsy, H\&E stain, $\times 200$ magnification

TelVysion $18 \mathrm{p}$ probe. All probes were supplied by Abbott Molecular, Des Plaines, IL, USA.

\section{Results including flow cytometry and cytogentetics}

The lymph node specimen was received fresh, measuring $1.2 \times 1.2 \times 0.7 \mathrm{~cm}$, and felt a bit firm grossly.

A portion of the fresh specimen was sent for cytogenetic analysis. Cytogenetic metaphase analysis of the lymph node was not performed due to lack of metaphase cells. FISH analysis demonstrated a loss of the p11.3 region of chromosome 18 using a subtelomere probe specific for the p11.3 region of chromosome 18 (yellow dot signal, Fig. 1a, left field). A probe for the p11.2 region of chromosome 18 was not available. With cytogenetic metaphase analysis of the BM specimen 4 years ago and UB specimen from last year, chromosomal karyotyping revealed a karyotype of 46, XX, del(18) (p11.2)[30]/46,XX [8] (i.e., a deletion of most of the short arm of chromosome 18; Fig. 1b).

FISH demonstrated normal signal patterns for chromosomes 5, 7, 11, and 21 in all nuclei from UB specimens from last year and 10 months ago.

Microscopically, the lymph node specimen showed distorted nodal architecture by an infiltrate of bizarre, giant cells (Figs. 2a and 3). While there was marked fibrosis, a significant component of tumoral spindle cell proliferation process was not seen. The giant cells showed nuclear lobulation with irregular chromatin distribution and abundant cytoplasm. Multinucleated giant cells were also seen. Abnormal mitotic activities were present. The dysplastic changes of the giant cells were so profound that a mesenchymal malignancy was considered the most likely diagnosis during our initial workup, even though the history of myelofibrosis was known. Most of the giant cells appeared larger than the dysplastic megakaryocytes on the marrow core biopsy sections from 4 years prior (Fig. 2b)
Fig. 3 a Lymph node biopsy, immunostain for vimentin, $\times 200$ magnification. b Lymph node biopsy, immunostain for cytokeratin, $\times 200$ magnification c Lymph node biopsy, immunostain for CD45, $\times 200$ magnification. d Lymph node biopsy, immunostain for CD61, ×200 magnification
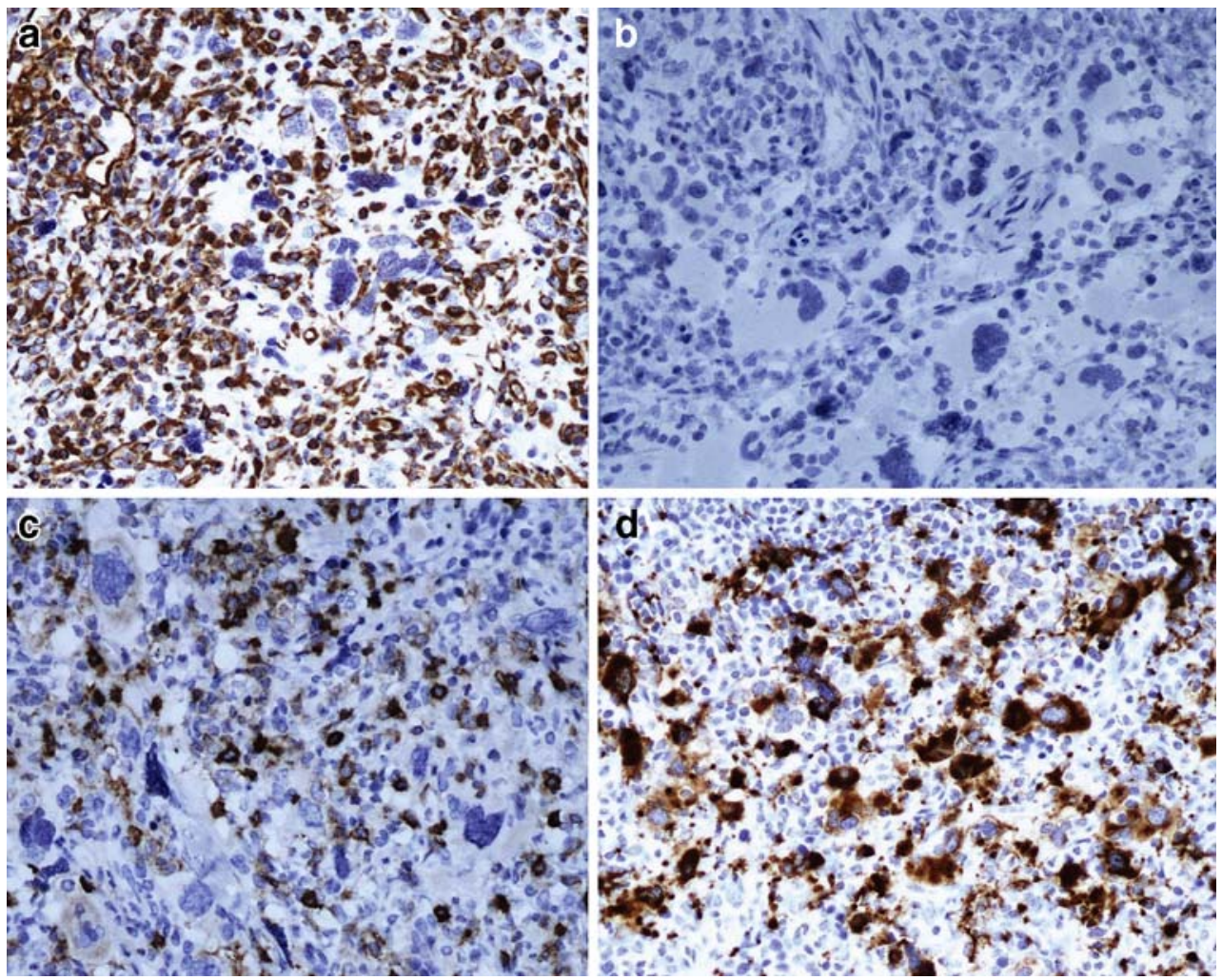
and did not show the hyperchromatic or pyknotic nuclei seen on the marrow. There were also scattered, large mononuclear cells that were smaller than the giant cells. These cells showed dysplastic and malignant-appearing nuclei. Other hematopoietic elements were not obvious. The dysplastic cells and the giant cells were negative for vimentin and cytokeratin by immunohistochemical stain (Fig. 3a, b). Vimentin stains the interstitial cells on the lymph node section. Not surprisingly, the giant cells were also negative for CD45 (Fig. 3c), CD3, and CD20 (data not shown). Immunohistochemical stain for CD61, a marker of megakaryocytes (Integrin beta 3, also called platelet glycoprotein IIIa), was added and showed strong cytoplasmic staining in most of the giant cells (Fig. 3d). A diagnosis of myelofibrosis involving lymph node with infiltrating dysplastic megakaryocytes was then made as a result of the findings. The case was later seen by an outside consultant who concurred with the diagnosis.

Flow cytometry analysis of the node indicated less than $1 \%$ myeloid blasts based on the light scatter characteristics and combination of CD34 and CD33 positivity (data not shown). Additionally, there was no evidence of monoclonal $\mathrm{B}$ cells, abnormal $\mathrm{T}$ cells, cytokeratin-positive cells, or CD30-positive cells (data not shown).

\section{Discussion}

To our knowledge, this was the first case of PMF associated with a deletion of part of the short arm of chromosome 18 (3) The cytogenetic and FISH studies revealed deletions of the short arm of chromosome 18 in bone marrow, unstimulated blood, and lymph node at various times. As the deletion of part of the short arm of chromosome 18 detected in the karyotypes from BM and UB extended from p11.2 to the terminus of the short arm, it represented a much larger area of the genome than the FISH probe covered. Therefore, the deletion in BM and UB cells was apparently the same as the deletion of p11.3 of chromosome 18 detected by interphase FISH in the lymph node. To our knowledge, deletions of chromosome 18 have not been reported to be associated with primary myelofibrosis. Numerical abnormalities of chromosomes 7, 8, and 9 and structural changes of $1 \mathrm{q}, 5 \mathrm{q}, 13 \mathrm{q}$, and $20 \mathrm{q}$ have been reported in primary myelofibrosis (4-6). The deletion of $18 \mathrm{p}$ seen in this patient may reflect the loss of one or more critical genes within the deleted region that could be associated with the disease state in this patient. Loss of homozygosity of chromosome $18 \mathrm{p} 11.3$ was reported in breast cancer, both invasive carcinoma and in situ ductal carcinoma (7). Amplification of the $18 \mathrm{p} 11.3$ band was seen in esophageal carcinoma (8). A putative tumor suppressor gene, DAL-1, was recently mapped to chromosome band
18 p11.3 (9). Cytogenetic abnormalities of chromosome $8 \mathrm{p} 11$ involving fibroblast growth factor receptor 1 (FGFR1) gene has been described in a subset of chronic myeloproliferative disorder to which primary marrow fibrosis belongs to (10). FISH of $8 \mathrm{p} 11$ has not been performed and may be pursued in the follow-up of the patient.

As far as the histopathologic diagnosis is concerned, it is clear that clinical history can provide important clues that lead to the correct diagnosis. In this case, without the history, it would have been much harder to include the myeloid metaplasia into the differential diagnosis. Secondly, both the lymph node and the bone marrow showed that megakaryocytes can be the predominant element of myeloid metaplasia in myelofibrosis. In addition, the megakaryocytes outside the marrow may be very dysplastic and have different morphological features from that in the marrow. It is possible that the marked fibrosis may have affected the morphology of megakaryocytes in the marrow. Myeloid metaplasia usually shows infiltration by multiple immature hematopoietic elements such as nucleated erythrocytes, early myeloid cells, and megakaryocytes. A correct diagnosis is often straightforward. However, if the process constitutes only or predominantly dysplastic megakaryocytes as in this case, it could be a diagnostic challenge. Immunohistochemistry was invaluable in the diagnosis of this case. The availability of specific antibodies helped to rule out certain differential diagnoses. CD61 antibody, which is a routinely used marker for megakaryocytes, helped to confirm the diagnosis.

In this patient, the finding of a small number of myeloblasts in the lymph node specimen by flow analysis was a hint for a possible myeloid process and led to appropriate workup. On the other hand, the number of myeloblasts was not high enough to suggest a blastic crisis.

Last, while there was no histological evidence, it was conceivable that the patient's pulmonary problems were related to circulating megakaryocytes plugging the capillaries in the lungs. This has been documented in the literature (11), especially when there were no other identifiable causes found in the pulmonary workup.

Conflict of Interest The authors declare that they have no conflict of interest.

\section{References}

1. Swerdlow SH et al (eds) (2008) World Health Organization Classification of Tumors. Pathology and genetics of tumors of hematopoietic and lymphoid tissues. IARC, Lyon

2. Tefferi A et al (2000) Myelofibrosis with myeloid metaplasia. New Engl J Med 342:1255-1265 
3. http://atlascytogeneticsoncology.org

4. Ahmed A, Chang CC (2006) Chronic idiopathic myelofibrosis: clinicopathologic features, pathogenesis and prognosis. Arch Pathol Lab Med 130:1133-1143

5. Bench AJ, Pahl HL (2005) Chromosomal abnormalities and molecular markers in myeloproliferative disorders. Semin Hematol 42:196-205

6. Lock G, Kendrick C (2006) Myelofibrosis with myeloid metaplasia. NZ J Med Lab Sci 60:3-6

7. Kittiniyom K et al (2001) Allelic loss on chromosome band 18 p 11.3 occurs early and reveals heterogeneity in breast cancer progression. Breast Cancer Res 3:192-198
8. Nakakuki K et al (2002) Novel targets for the 18p11.3 amplification frequently observed in esophageal squamous cell carcinoma. Carcinogenesis 23:19-24

9. Tran YK et al (1999) A novel member of the NF2.ERM/4.1 superfamily with growth suppressing properties in lung cancer. Cancer Res 59:35-43

10. Goradia A et al (2008) The 8p11 myeloproliferative syndrome: review of literature and an illustrative case report. Int J Clin Exp Pathol 1:448-456

11. Hill G et al (2008) Pulmonary hypertension as a consequence of alveolar capillary plugging by malignant megakaryocytes in essential thrombocythaemia. Intern Med J 26:852-853 